stration talks for boys and girls throughout the school year, in collaboration with the science teachers, at which large-scale experiments are shown which cannot easily be staged with school resources. An approximate estimate of the cost, based on the proportion of overhead charges allocated to the

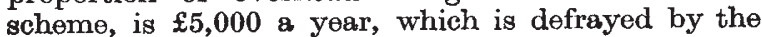
generous assistance of industrial firms and other bodies which are interested in the venture. Some 16,000 tickets are issued annually. The young people come from an area which includes about one-quarter of the schools in Englend, and their keenness indicates that this is a very repaying way of using the resources. Some half-dozen such centres operating on a similar whole-time basis would cover most of Great Britain. Certain famous annual lectures sponsored by various bodies are by tradition the occasion for numerous carefully prepared experiments, and these lectures draw enthusiastic audiences. Television can, of course, be a powerful means of transmission; but the laboratory resources must still be there behind the scenes for providing the programmes. My contention, therefore, is that merely increasing the number of talks and articles about science will not get us very far in interesting the general public, that on the other hand one gets the most rewarding response if one stages shows of experiments for them, and that if their interest is first so aroused, they will be much more able" to appreciate the talks and articles.

\title{
OBITUARIES
}

Prof. D. R. Hartree, F.R.S.

Prof. D. R. Hartree, who died suddenly in Cambridge on February 12 at the age of sixty, had been Plummer professor of mathematies in the University since 1946. Before that he had spent fifteen years in Manchester, first as Beyer professor of applied mathematics and later as professor of theoretical physics. He was elected a Fellow of the Royal Society in 1932.

Hartree was born in Cambridge in 1897 and was seventeen years old when the First World War broke out. He served in the Munitions Invention Department and held a commission in the R.N.V.R. When the War ended, he returned to Cambridge to teke the Natural Sciences Tripos. He became a Fellow of his own College, St. John's, and later migrated to Christ's College, where he held a fellowship for a short time before going to Manchester.

Some of Hartree's earliest work was on the propagation of radio waves in a magneto-ionic medium, and he wrote several important papers on this subject. His principal contribution to physics, however, was the development of the method of self-consistent fields for the computation of atomic wave functions. Early applications of wave mechanics had been to simple atoms such as those of hydrogen and helium, but Hartree saw a way of obtaining, by numerical means, wave functions for heavier atoms. He exploited this method with great vigour, and a long series of papers appeared in which Hartree and his collaborators presented the results of calculations for a large number of atoms.

Hartree's work on atomic wave functions led him to a more general interest in numerical analysis, and this eventually became his major preoccupation. To him numerical analysis was above all a practical subject and he brought the same kind of enthusiasm to computing as others bring to practical work in a laboratory. He always liked to tackle a new problem, and anyone who brought a problem to him would, as like as not, be given practical help as well as advice.

Although Hartree enjoyed computing-a little while before his death he claimed that he had some 10,000 hours of personal computing to his credithe appreciated very early the advantages which might be obtained from mechanical aids. He was responsible for introducing the differential analyser into Britain, and the machine which, at his instance, was installed in the University of Manchester in 1935 was used to solve a wide range of problems. During the Second World War the machine was occupied with military problems of various kinds including, notably, an investigation into the mode of operation of the radar magnetron.

When the War ended, Hartree visited the United States to study the developments then going on in automatic digital computers. His practical experience of numerical analysis enabled him to make a distinctive contribution in this field and he rapidly became accepted as an authority. He was much in demand as a lecturer, and a course he delivered at the University of Illinois in the autumn of 1948 was published in book form.

When Hartree arrived in Cambridge in 1946, development work on an electronic digital computer was starting in the Mathematical Laboratory. Hartree associated himself with this project with characteristic enthusiasm and the Laboratory owes much to him for encouragement, advice and practical assistance. He continued his close association with the Laboratory until his death and took a prominent part in the running of successive summer schools in programme design and in the teaching for the diploma in numerical analysis and automatic computing.

Hartree did much to advence the interests of the Cavendish Laboratory, where he had his office. In particular, he was largely instrumental in reorganizing and improving the teaching of mathematics to science students, and in establishing theoretical physics as an optional subject in Part 2 of the Natural Sciences Tripos. In addition to his scientific interests, Hartree was a very keen musician, and played an important part in the founding of the Faculty of Music in the University of Manchester.

Hartree will be greatly missed by his many friends. $\mathrm{He}_{\theta}$ was the kindest and most unassuming of men, and endeared himself to all who worked with him. M. V. WHKES

\section{Dr. C. J. Davisson}

Prystcists all over the world, and especially those of the older generation, will have heard with deep regret of the death on February 1 of C. J. Davisson.

Davisson is known best for his work on the diffraction of electrons by metal crystals, which established for the first time the wave character of free electrons and proved L. de Broglie's relation $\lambda=h / p$ between the wave-length $\lambda$ and the momentum $p$ of an electron. In fact, this work was the outcome of a long series of experiments started for quite a different purpose. 